\title{
HER2 as a therapeutic target in the gastric cancer: is it sufficient?
}

\author{
Rupesh Chaturvedi • Amar B. Singh
}

Received: 17 December 2011 / Accepted: 12 June 2012 /Published online: 5 July 2012

(C) Indian Society of Gastroenterology 2012

Globally, gastric adenocarcinoma is the second leading cause of cancer-related deaths, with the highest incidence reported in northeast Asia [1]. According to the World Health Organization (WHO) 2008 report, gastric cancer affects approximately one million people every year. Ironically, symptoms of gastric cancer are often mistaken for other gastric diseases, and therefore many patients are diagnosed with the cancer at an inoperable or metastatic stage. Thus, despite the improvement of surgical techniques and the recent availability of new chemotherapy regimens, outcome of the patients with clinically advanced disease is usually poor. The overall 5 -year relative survival for 2001-2007 in USA was $26.3 \%$ [2]. Therefore, new treatment options are needed on an urgent basis.

Gastric carcinogenesis is a very complex biological process and gastric tumors are heterogeneous in nature. Two histologically distinct categories of gastric tumors are reported: (1) a diffuse-type tumor consisting primarily of the individually infiltrating neoplastic cells that do not form glandular structures, and (2) a more common intestinal-type tumor, which progresses through a series of histological changes (normal mucosa to trophic gastritis to intestinal metaplasia to dysplasia and to adenocarcinoma). A better understanding of the mo-

R. Chaturvedi $(\square)$

Division of Gastroenterology, Hepatology, and Nutrition, Department of Medicine,

Vanderbilt University School of Medicine,

1030C MRB IV, 2215B Garland Ave,

Nashville, TN 37232-0252, USA

e-mail: rupesh.chaturvedi@vanderbilt.edu

A. B. Singh

Department of Surgery, Vanderbilt University Medical Center,

Nashville, TN, USA lecular and signaling processes that regulate growth and progression of the gastric cancer will not only help develop new biomarkers for the risk assessment but may also help accelerate the design of new molecular-targeted therapies with the potential of interfering with the signaling cascades involved in cell differentiation, proliferation, and survival of the gastric cancer cells. Moreover, the classification of patients on a molecular basis is essential to maximize the benefit of targeted agents and to avoid significant toxicity.

The family of the epidermal growth factor receptor tyrosine kinases (EGFRs; Human Epidermal Growth Factor Receptor [HERs]) is one of the key regulators of oncogenic transformation and tumor progression, and is composed of four members: HER1 (also known as the EGFR), HER2, HER3 (also termed Erythroblastic leukemia viral oncogene homolog: ErbB-3), and HER4 (also termed ErbB-4) [3-5]. All four receptors are structurally similar and consist of an extracellular ligand-binding domain, a helical transmembrane segment, and an intracellular protein tyrosine kinase domain. Binding of a ligand to the receptor initiates homo and/or heterodimerization and subsequent tyrosine kinase activation [3]. The tyrosine kinase activation then stimulates an intracellular signaling cascade including PI3-kinase and mitogen-activated protein (MAP) kinase that modulate cellular responses to diverse stimuli, potentially through the modulation of gene expression associated with the key cellular processes, including cell survival and proliferation (Fig. 1) [6-8]. However, HER3 lacks the tyrosine kinase activity, and a ligand for HER2 still remains to be found [9].

The expression of the EGFR family of receptors is frequently altered in gastric cancers, primarily due to gene amplification [10]. In fact, the importance of HER2 as a key regulator of gastric cancer is evident by the fact that on 28 January 2010, European authorities approved trastuzumab, 
a monoclonal antibody against HER2, in combination with chemotherapy for first-line treatment of patients with HER2positive advanced gastric cancer. HER2 overexpression has been observed in $9 \%$ to $38 \%$ of gastric cancer cases as evaluated by immunohistochemistry (IHC) using polyclonal antibodies directed against various domains and has been implicated specifically in the intestinal type of gastric cancers [11]. Similar rates of HER2 expression have been reported using monoclonal antibodies and/or by detecting gene amplification using fluorescent in situ hybridization (FISH) analysis [12]. In fact, a careful review of the published literature suggests that IHC can be reasonably used as the primary screening method to evaluate the status of HER2 expression, and trastuzumab can serve as a therapeutic agent to treat gastric cancer patients with high HER2 expression levels [12].

In the current issue, Sekaran et al. concluded that HER2 expression is upregulated in half of the advanced gastric carcinoma patients [13]. But they did not observe an association between levels of HER2 expression and demographic or histo-clinical parameters. However, a large prospective study including more patients from different regions of India will be needed to clearly understand the role of HER2 in the regulation of gastric cancer in India. It will also be important to include a cohort of patients, in the above analysis, with preneoplastic lesions such as atrophic gastritis, metaplasia, and dysplasia to understand whether HER2 upregulation is an early event in the process of gastric carcinogenesis and to examine its correlation with cancer progression.

Also, it needs to be cautioned that the upregulation of HER2 does not necessarily represent a definitive selection parameter for treatment, as not all HER2-positive patients respond to the trastuzumab treatment. This represents a great challenge for the selection of patients for anti-HER2 treatment. Therefore, molecular mechanisms for gastric tumor heterogeneity and the inherent complex regulation of signaling networks should be considered for the selection of patients for HER2-targeted therapy, as: (1) Higher levels of HER2 expression in an individual may render the amount of available antibody insufficient to effectively block the target. (2) The constitutive activation of downstream signaling; for example, amplification of PI3K signaling due to the loss of lipid phosphatase PTEN or expression of PIK3CA-activating mutations is associated with lower response to trastuzumab in HER2-positive breast cancer patients [14, 15]. (3) The compensatory upregulation of alternative or redundant parallel signaling cascade can also provide a resistance to trastuzumab treatment. In this regard, lessons can be learned from the breast cancer setting, where some patients develop resistance over time despite an initial response to trastuzumab. Of interest, it has been reported that in HER2positive breast cancer patients, overexpression of EGFR and/or HER3 associates with a poor response to trastuzumab therapy [16]. Notably, in vitro trastuzumab can block the

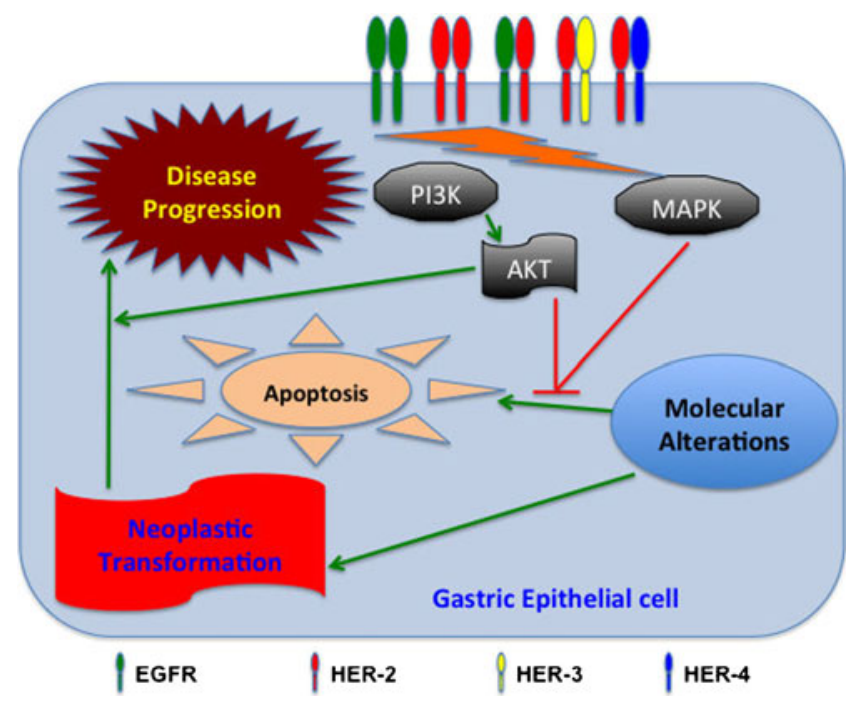

Fig. 1 Signaling cascades regulated by the four HER family members -EGFR, HER2, HER3, and HER4. Different ligands bind to the extracellular domain of the receptors, inducing homodimerization or heterodimerization to form several receptor combinations. Receptor phosphorylation resulting from the tyrosine kinase activity within the cytoplasmic domains activates the PI3K/AKT axis and the MAPK and inhibits apoptosis in epithelial cells with molecular alterations, which leads to neoplastic transformation. Downstream signaling molecules also increase cell-cycle progression and proliferation, which results in disease progression. EGFR, epidermal growth factor receptor; HER, human epidermal growth factor receptor; PI3K, phosphatidylinositol 3kinase; MAPK, mitogen-activated protein kinase

activation of HER2 homodimer, but not HER2/EGFR or HER2/HER3 heterodimer. (4) HER2-positive breast tumors with high levels of HER2 C-terminal fragments that lack the trastuzumab-binding epitope have also been reported to be resistant to trastuzumab [17]. Notably, the above described molecular alterations have not yet been examined in HER2-positive gastric tumors, and thus a retrospective study is needed to evolve comprehensive and precise biological signatures using next generation omics technology, which can be validated in individual patients for a more personalized treatment.

\section{References}

1. Jemal A, Bray F, Center MM, Ferlay J, Ward E, Forman D. Global cancer statistics. CA Cancer J Clin. 2011;61:69-90.

2. Polk DB, Peek RM Jr. Helicobacter pylori: gastric cancer and beyond. Nat Rev Cancer. 2010;10:403-14.

3. Yarden Y, Sliwkowski MX. Untangling the ErbB signalling network. Nat Rev Mol Cell Biol. 2001;2:127-37.

4. Hynes NE, Lane HA. ERBB receptors and cancer: the complexity of targeted inhibitors. Nat Rev Cancer. 2005;5:341-54.

5. Schlessinger J. Ligand-induced, receptor-mediated dimerization and activation of EGF receptor. Cell. 2002;110:669-72.

6. Hsieh AC, Moasser MM. Targeting HER proteins in cancer therapy and the role of the non-target HER3. Br J Cancer. 2007;97:453-7. 
7. Holbro T, Civenni G, Hynes NE. The ErbB receptors and their role in cancer progression. Exp Cell Res. 2003;284:99-110.

8. Carpenter G, Cohen S. Epidermal growth factor. J Biol Chem. 1990;265:7709-12.

9. Baselga J, Swain SM. Novel anticancer targets: revisiting ERBB2 and discovering ERBB3. Nat Rev Cancer. 2009;9:463-75.

10. Tokunaga A, Onda M, Okuda $T$, et al. Clinical significance of epidermal growth factor (EGF), EGF receptor, and c-erbB-2 in human gastric cancer. Cancer. 1995;75:1418-25.

11. Bang YJ, Van Cutsem E, Feyereislova A, et al. Trastuzumab in combination with chemotherapy versus chemotherapy alone for treatment of HER2-positive advanced gastric or gastrooesophageal junction cancer (ToGA): a phase 3, open-label, randomised controlled trial. Lancet. 2010;376:687-97.

12. Boers JE, Meeuwissen H, Methorst N. HER2 status in gastrooesophageal adenocarcinomas assessed by two rabbit monoclonal antibodies (SP3 and 4B5) and two in situ hybridization methods (FISH and SISH). Histopathology. 2011;58:383-94.
13. Sekaran A, Kandagaddala RS, Darisetty S, et al. HER2 expression in gastric cancer in Indian population - an immunohistochemistry and fluorescence in situ hybridization study. Indian J Gastroenterol. 2012;31. doi:10.1007/s12664-012-0214-0.

14. Nagata $Y$, Lan KH, Zhou X, et al. PTEN activation contributes to tumor inhibition by trastuzumab, and loss of PTEN predicts trastuzumab resistance in patients. Cancer Cell. 2004;6:117-27.

15. Berns K, Horlings HM, Hennessy BT, et al. A functional genetic approach identifies the PI3K pathway as a major determinant of trastuzumab resistance in breast cancer. Cancer Cell. 2007;12:395-402.

16. Ritter CA, Perez-Torres M, Rinehart C, et al. Human breast cancer cells selected for resistance to trastuzumab in vivo overexpress epidermal growth factor receptor and ErbB ligands and remain dependent on the ErbB receptor network. Clin Cancer Res. 2007;13:4909-19.

17. Scaltriti M, Rojo F, Ocana A, et al. Expression of p95HER2, a truncated form of the HER2 receptor, and response to anti-HER2 therapies in breast cancer. J Natl Cancer Inst. 2007;99:628-38. 